\title{
Speciation of Candida using CHROMagar isolated from Various Clinical Samples
}

\author{
Shwetha $\mathrm{DC}^{\mathbf{1}}{ }^{*}$, Venkatesha $\mathrm{D.}^{\mathbf{2}}$ \\ DOI: https://doi.org/10.17511/jopm.2020.i04.06 \\ 1* Shwetha D C, Assistant Professor, Department of Microbiology, Adichunchanagiri Institute of Medical Sciences, B G Nagara, Karnataka, \\ India. \\ 2 Venkatesha D., Professor and Head, Department of Microbiology, Adichunchanagiri Institute of Medical Sciences, B G Nagara, Karnataka, \\ India.
}

Introduction: Infections due to Candida especially Non-albicans Candida (NAC) species are increasingly being reported in recent years. Isolation and identification up to species level are of utmost importance in the early management of these infections. Materials and methods: All the clinical samples received in the Microbiology laboratory were inoculated on to Blood agar and MacConkey agar. The isolates which revealed Gram-positive budding yeast cells on Gram staining were further inoculated on to SDA (Sabouraud Dextrose agar) and Hicrome Candida differential agar. Candida was differentiated into species based on color and morphology on Hicrome agar. Result: A total of 126 Candida were isolated from various clinical samples. C.Albicans was the most common species isolated $(49.2 \%)$ followed by C. tropicalis $(19.8 \%)$, C. glabrata $(19 \%)$, and C. krusei $(12 \%)$.Conclusion: Hicrome agar is a simple, rapid, and cost-effective medium for the speciation of Candida.

Keywords: Candida albicans, Hicrome agar, Non-albicans Candida

Corresponding Author

Shwetha D C, Assistant Professor, Department of Microbiology, Adichunchanagiri Institute of Medical Sciences, B G Nagara, Karnataka, India. Email: shwetha.dasarahalli@gmail.com
How to Cite this Article

To Browse

Shwetha DC, Venkatesha D. Speciation of Candida using CHROMagar isolated from Various Clinical Samples. Trop J Pathol Microbiol. 2020;6(4):303308.

Available From

https://pathology.medresearch.in/index.php/jopm/ar ticle/view/448

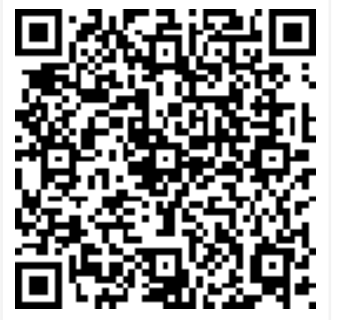

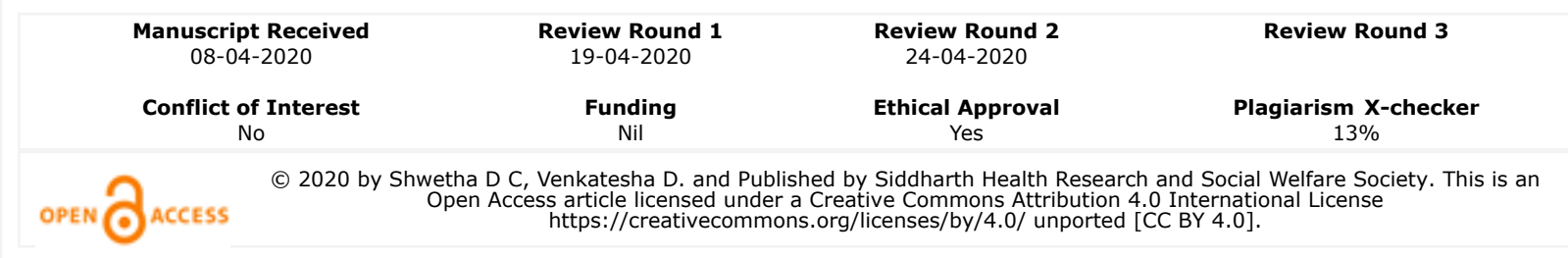




\section{Introduction}

Over the last few years, the incidence of mycotic infections has progressively increased. Fungi once considered as nonpathogenic or less virulent are now recognized as a primary cause of morbidity and mortality in immunocompromised and severely ill patients [1].

Candida species belong to the normal microbiota of an individual's mucosal oral cavity, gastrointestinal tract, and vagina and are responsible for various clinical manifestations from simple mucocutaneous overgrowth to invasive infections like bloodstream infections, which is due to their great adaptability to different host environment [2].

In the 1980s C.Albicans accounted for more than $80 \%$ of all Candida isolates recovered from yeast infections. More recently Non-albicans Candida (NAC) species have been recovered with increasing frequency [3] Isolation and prompt identification of the infectingorganism to the species level is essential to optimize the earlyantifungal therapy as certain species like $C$. kruseiare inherently resistant to azole drugs $[4,5]$.

The longer turnaround time taken by conventional methods of identification makes them less popular among the clinicians as early diagnosis is essential for initiating appropriate therapy. In order to facilitate rapid identification, several chromogenic substrates containing culture media have been developed. Hicrome agar is a differential media that allows selective isolationof yeasts and identifies colonies of C. Albicans, C. glabrata, C. krusei, and C. tropicalis[6].

The present study was undertaken to determine the prevalence of Candida species among various clinical samples.

\section{Materials and Methods}

Thiscross-sectionalstudy was conducted in the Department of Microbiology of a Tertiary care hospital situated in a rural setup. Periodic sampling was done for a period of 7 months from April 2019 to October 2019.

Inclusion criteria- The study includes Candida isolates from various clinical samplessent routinely to the Microbiology laboratory

Exclusion criteria-Patients on any antifungal therapy 6 weeks prior to sample collection were
Excluded from the study.

This present study was done after obtaining the Institutional ethical committee clearance.

All samples were collected using aseptic precautions. After receiving in the Microbiology laboratory, the samples were inoculated onto both Blood agar and MacConkey agar, and plates were incubated at $370 \mathrm{C}$ for $24-48$ hours aerobically. Colonies that appearedsmooth, pasty, opaque, white, or beige were suspected asCandidacolony (Figure 1)and Gram stain was done for confirmation (Figure 2).

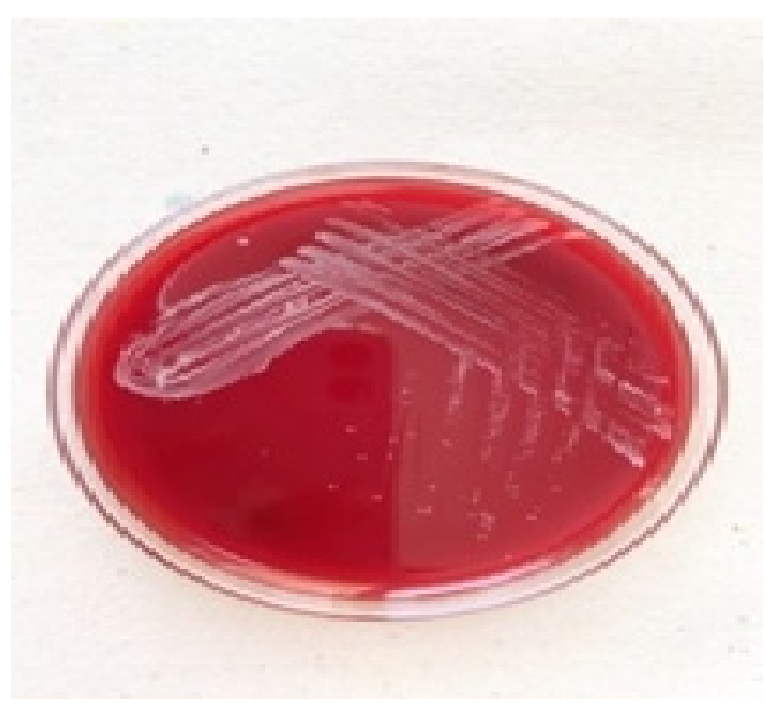

Fig-1: Candida on Blood agar.

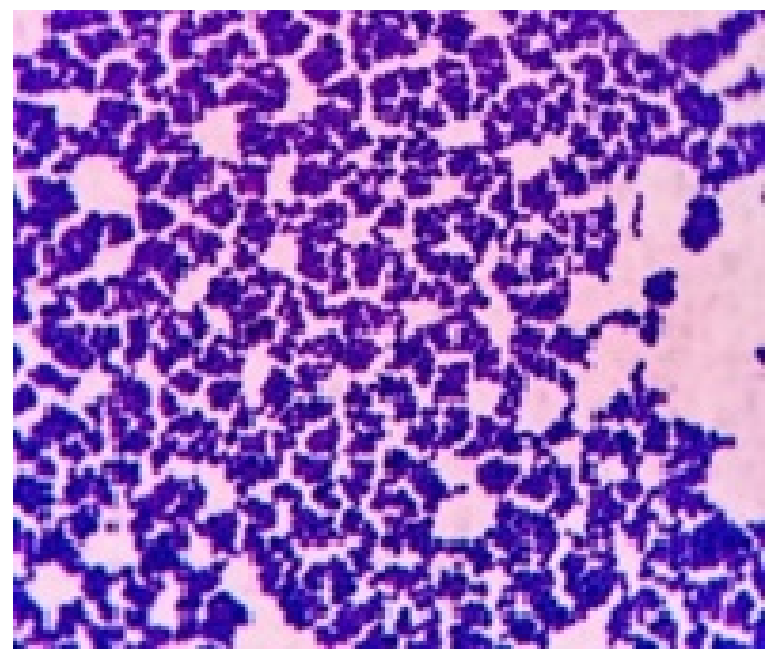

Fig-2: Gram stain showing Candida.

The isolate that revealed gram-positive budding yeast cells was inoculated ontoSabouraud Dextrose agar(Figure 3)and Hicrome Candida differential agar. 


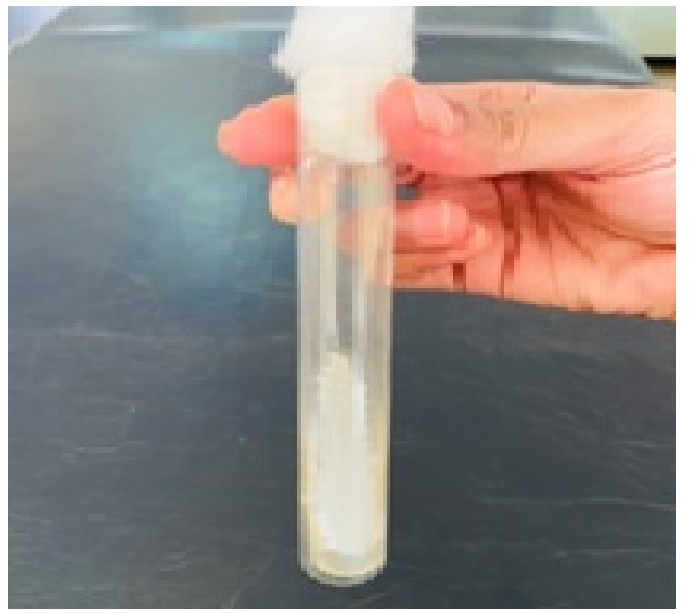

Fig-3: Candida growth on Sabouraud Dextrose agar.

The growth obtained on SDA was further subjected to Gram stain and germ tube test. Germ tube test was done to differentiate C.Albicans and C.dublinenses from other Candida species. An isolated colony of Candidawas suspended in $0.5 \mathrm{ml}$ of serum and was incubated at $37^{\circ} \mathrm{C}$ for 3 hours. A drop of this suspension was placed on a microscope slide and examined for the presence of germ tubes[7]. Speciation of the Candida isolates was done by inoculating it on Hicrome Candida differential agar.Hicrome agar was prepared as per the manufacturer's instructions and incubated at $300 \mathrm{c}$ for 24-48 hours aerobically. Species identification was done by the morphology and color of the colony. C.Albicansproduce light green colonies, C. tropicalismetallic blue colonies, C.kruseiproduces purple fuzzy colonies, and C. glabrata white to cream-colored colonies (Figure 4).

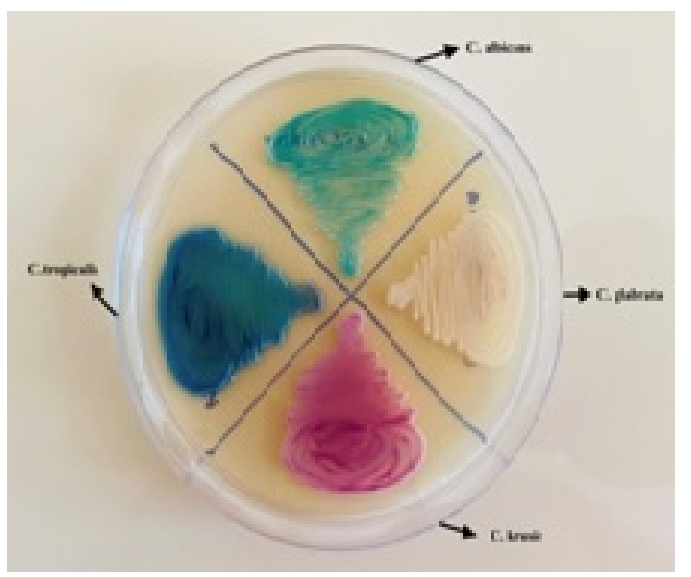

\section{Fig-4: Candida species on CHROM agar.}

The isolates that remained doubtful in their appearanceon Hicromewas considered as unidentified and excluded from the study. Candida isolates from clinical specimens like oropharyngeal,
Vaginal, urinary, and bronchial secretions were considered significant only after repeated isolation. Descriptive statistics such as proportion and frequency were used to interpret the results.

\section{Results}

A total number of 126 Candida were isolated from various clinical samples. All isolates grew well on Hicrome Candida differential agar after 48 hours of incubation at $30^{\circ} \mathrm{C}$. Most of the isolates were from High vaginal swab $(n=78)$ followed by Urine $(n=36)$, Sputum $(n=04)$, Pus $(n=04)$, Blood $(n=02)$, Cerebrospinal fluid $(n=01)$ and Endo Tracheal Tube $(n=01)$ respectively (Table 1$)$.

Table 1: Distribution of Candida species.

\begin{tabular}{|l|l|l|l|l|l|}
\hline \multirow{2}{*}{ Sample } & \multicolumn{4}{c|}{ Candida species } & \multirow{2}{*}{ Total } \\
\cline { 2 - 5 } & C. albicans & $\begin{array}{l}\text { C. } \\
\text { tropicalis }\end{array}$ & $\begin{array}{l}\text { C. } \\
\text { glabrata }\end{array}$ & C. krusei & \\
\hline High Vaginal swab & 39 & 12 & 18 & 09 & $78(61.9 \%)$ \\
\hline Urine & 16 & 10 & 06 & 04 & $36(28.6 \%)$ \\
\hline Pus & 04 & 00 & 00 & 00 & $04(3.2 \%)$ \\
\hline Sputum & 01 & 03 & 00 & 00 & $04(3.2 \%)$ \\
\hline Blood & 01 & 00 & 00 & 01 & $02(1.5 \%)$ \\
\hline $\begin{array}{l}\text { Cerebro spinal } \\
\text { fluid }\end{array}$ & 01 & 00 & 00 & 00 & $01(0.8 \%)$ \\
\hline Endotracheal tube & 00 & 00 & 00 & 01 & $01(0.8 \%)$ \\
\hline Total & $\begin{array}{l}62 \\
(49.2 \%)\end{array}$ & $\begin{array}{l}25 \\
(19.8 \%)\end{array}$ & $24(19 \%)$ & 15 & $126(100 \%)$ \\
& & & & $(12 \%)$ & \\
\hline
\end{tabular}

Out of 126 Candida isolates, C. Albicanswas the most common species in $62(49.2 \%)$ strains. The remaining $64(50.8 \%)$ strains showed Non-albicans Candida. Out of 64 Non-albicans Candidaisolates, 25 were C. tropicalis(19.8\%), 24 were C. glabrata(19\%) and 15 were C. krusei (12\%) respectively.

Note: Out of 78 isolates from High Vaginal Swab (HVS), the most common species was C. Albicans followed by C. glabrata, C. tropicalis, and C. krusei. Among the 36 urine samples, the most common species was $C$. Albicans followed by $C$. tropicalis, $C$. glabrata, and C. krusei. Species wise distribution of Candida isolates from various clinical samples is shown in Table 1.

\section{Discussion}

Identification of Candida strains to the species level is increasingly necessary because of their variation both in their ability to cause infection and also in their susceptibility to antifungal agents. Specieslevel of yeast identification is mandatory for epide- 
Miological purpose and laboratory diagnosis[8].Hicrome Candida differential agar medium accurately identifies the important Candida species namely C. Albicans, C. tropicalis, $C$. glabrata, C. dubliniensis, and C. krusei based on their color and morphological features [9].

Fromthe present study, the rate of isolation of NAC was $50.8 \%$ and the isolation rate of C.Albicans was 49.2\%. Non-albicans Candidapredominated over C

Albicans which correlates well with many other studies. In a study done by Kaup $S$ et al[10], the Non-albicans Candida accounted for $50 \%$ of the isolates and the commonest species was C. Albicans (50\%) followed by C. tropicalis (27.08\%). Nonalbicans Candida(52.8\%) isolates were more than C. Albicans(47.2\%) in a study by Samyuktha AA et al[11]Predominance of Non-albicans Candida(54\%) over C. Albicans(46\%) was also shown in a study by Madhumathi $B$ et al[12].The present study also correlated with the study done by Marak MB et al [13] where the rate of isolation of Non-albicans Candida was $54.5 \%$.

Though Candida albicans is considered to be the commonest species causing human diseases, there is a striking increase in the isolation rate ofNonalbicans Candidaspecies, primarilyCandida tropicalis, Candida glabrata, Candida krusei, and Candida parapsilosis. This rise in Non-albicans Candida species has been associated with significant morbidity and mortality [14]. Hence, identification of Candida upto species level becomes necessary for the initiation of early and effective therapy. As NAC species significantly vary in their prevalence among different countries and health-care setups within a country, species identification plays an important role in the formulation of local therapeutic guidelines [15].

In routine diagnostic laboratories, Sabouraud dextrose agar (SDA) iswidely used for the isolation of all yeast species from a clinical specimen. Sabouraud dextrose agar is not a differential medium, and multiple yeast growth cannot be easily distinguished from each other. In the majority of the laboratories germ tube test is used to differentiate C. Albicans and C. dubliniensis from other Candida species. Although it is a rapid test, it may lead to false positive and false negative results [16].The other conventional methods like sugar fermentation and sugar assimilation tests used for the speciation of Candidaare very time consuming and cumbersome. Molecular methods are very expensive
And available only at reference centers.

Chromogenic agar is a rapid method to differentiateCandidaspecies asit contains enzymatic substrates that are linked to chromogenic compounds. When a specific enzyme cleaves the substrate, the chromogenic substances produce color. The action of different enzymes produced by yeast species results in color variation which is useful for the presumptive identification of some

Yeasts [14]. Another important advantage of chromogenic medium is it greatly facilitates the detection of specimens containing a mixture of yeast species though there were no mixed cultures in the present study. The prompt detection of such clinical scenarios of multiple yeast etiology may be an aid for early appropriate treatment decisions [16].

In the present study, amongst the NACmost frequently isolated species was C.tropicalis followed by $C$. glabrata and C. krusei. Many other studies have also shown the preponderance of $C$. tropicalis over other NACspecies $[17,18,19,20,21,22,23]$.

\section{Limitations of the study}

Conventional methods for the identification of Candida species like sugar fermentation and assimilation tests are not included in the present study. Hence, other species of Candida like $C$. guilliermondii, C. parapsilosis, C. kefyr could not be identified in the present study.

\section{Conclusion}

Identification of Candida up to species level is very important in the early management of Candidiasis. Non-albicans Candida species are increasingly associated with invasive Candidiasis and differ from C. Albicans with respect to epidemiology and antifungal susceptibility. The present study highlights the fact thatNAC has emerged as an important cause of infections even in our set up and can no longer be ignored as non-pathogens and contaminants.

\section{What does the study add to the existing knowledge?}

The current study results are also important for local monitoring of different Candida species which also helps in planning empirical therapies. Hicrome agar is a simple, rapid, and inexpensive method for 
The identification of Candida species and is suitable for laboratories with limited resources. The major pathogenic species like C. Albicans, C. tropicalis, C. glabrata, and C. kruseiare easily differentiated by their color and colony morphology within a short time.

\section{Author's contribution}

Dr. Shwetha D C.: Contributed for Study design, Literature search, Data collection, statistical analysis, manuscript preparation, editing, and review

Dr. Venkatesha D.: Contributed for Study design, Smanuscript preparation, editing and review

\section{Reference}

01. Deorukhkar SC, Saini S, Mathew S. Nonalbicans Candida infection- An Emerging Threat. Interdisciplinary Perspectives on Infectious Diseases. 2014.

doi: [Article:http://dx.doi.org/10.1155/2014/615958] [Crossref]

02. Sardi JCO, Scorzoni L, Bernardi T, et al. Candidaspecies-current epidemiology, pathogenicity, Biofilm formation, natural antifungal products and new therapeutic options. J Med Microbiol. 2013;62(1)10-24.

doi:

[Article:http://dx.doi.org/10.1099/jmm.0.045054-0] [Crossref]

03. Samyuktha AA, Saikumar C. Isolation, Identification and Speciation of Candida Species from Various Clinical Specimens in a Tertiary Care Hospital in Chennai. Scholar J App Med Sci. 2017;5(8F)3460-3468.

doi: [Article:http://dx.doi.org/10.21276/sjams] [Crossref]

04. Manjunath V, GS Vidya, Sharma A, Prakash MR, Murugesh. Speciation of Candida by HiCrome agar and Sugar assimilation test in both HIV infected and non-infected patients. Int J Biol Med Res. 2012;3(2)1778-1782.

[Crossref]

05. Jayachandran AL, Katragadda R, Ravinder T, Vajravelu L, Manoranjan L, Hemalatha $S$. Antifungal Susceptibility Pattern among Candida species- An Evaluation of Disc Diffusion and Broth Micro-dilution Method. J Microbiol Infect Dis. 2018;8(3)97-101.

doi: [Article:https://doi.org/10.5799/jmid.458457] [Crossref]
06. Shettar SK, Patil AB, Nadagir SD, Shepur TA, Mythri BA, Gadadavar S. Evaluation of HiCrome differential agar for speciation of Candida. J Acad Med Sci. 2012;2(3)102-104.

doi: [Article:https://doi.org/10.4103/22494855.132950][Crossref]

07. Forbes BA, Sahm DF, Weissfield AS. Laboratory methods in basic Mycology Bailey and Scott's diagnostic Microbiology. 12th edition, USAMosby Elsevier. 2007;629-716.

[Crossref]

08. Sankari SL, Mahalakshmi K, Kumar VN. Chromogenic medium versus PCR-RFLP in the speciation of Candida- A comparative study. BMC Research Notes. 2019;12;681.

doi: [Article:https://doi.org/10.1186/s13104-0194710-5][Crossref]

09. Amit Bhosale, S J Ghosh, P G Shadija. Identification of various Candida species by using CHROMagar Candida- A rapid screening method. Int J Rec Trends Sci Technol. 2015;16(3)596-598.

[Crossref]

10. Soumya Kaup, Jaya Sankarankutty, $H$ VBalasubramanya, Suma Kulkarni, M Nirmala. Speciation of Candida using HiChrome Candida Differential Agar. Int J Curr Microbiol App Sci. 2016;5(7)267-274.

doi:

[Article:http://dx.doi.org/10.20546/ijcmas.2016.507. 027][Crossref]

11. Madhumathi B, Rajendran R. Evaluation of Chrome Agar in Speciation of Marak MB, Dhanashree B. Antifungal Susceptibility and Biofilm Production of Candida Spp Isolated from Clinical Samples. Int J Microbiol. 2018.

doi: [Article:https://doi.org/10.1155/2018/7495218] [Crossref]

12. Dharmeshwari T, Chandrakesan SD, Mudhigeti $\mathrm{N}$, Perticia A, Kanungo R. Use of Chromogenic Medium for Speciation of Candida isolated from Clinical Specimens. Int J Cur Res Rev. 2014;6(1)1-5.

[Crossref]

13. Kumar S, Vyas A, Kumar M, Mehra SK. Application of CHROMagar Candida for Identification of Clinically Important Candida species and their Antifungal Susceptibility Pattern. Int J Biol Med Res. 2013;4(4)36003606.

[Crossref] 
14. Candida Species from Various Clinical Samples in a Tertiary Care Hospital. Int JCurr Microbiol App Sci. 2015;4(9)463-472.

[Crossref]

15. Vijaya D, Harsha TR, Nagaratnamma T. Candida Speciation Using Chom Agar. J Clin Diagnos Res. 2011;5(4)755-757.

[Crossref]

16. Deorukhkar SC, Roushani S. Identification of Candida Species- Conventional Methods in the Era of Molecular Diagnosis. Ann Microbiol Immunol. 2018;1(1)1002.

[Crossref]

17. Saxena N, Maheshwari D, Dadhich D, Singh S. Evaluation of Congo Red Agar for Detection of Biofilm Production by Various Clinical Candida Isolates. JEvol Med Dent Sci. 2014;3(59)1323413238.

doi:[Article:https://doi.org/10.14260/jemds/2014/ 3761][Crossref]

18. Jangla SM, Naidu R, Patel SC. Speciation and antifungal susceptibility testing of Candida isolates in various clinical samples in a tertiary care hospital in Mumbai. Int J Biomed Res. 2018;9(3)106-111.

doi: [Article:https://doi.org/10.7439/ijbr.v9i3.4678] [Crossref]

19. Rao RP. Isolation, identification and speciation of candida species from various clinical samples in a tertiary care teaching hospital in Karnataka, India. J Evid Based Med Health. 2019;6(11)866868.

doi:[Article:https://doi.org/10.18410/jebmh/

2019/182][Crossref]
20. B VigneshKanna, G Amar Kumar, M Swapna, Easow JM. Isolation and identification of Candida species from various clinical samples in a tertiary care hospital. Int J Res Med Sci. 2017; 5(8)3520-3522.

doi:[Article:http://dx.doi.org/10.18203/2320-

6012.ijrms20173554][Crossref]

21. Das KH, Getso M. Distribution of Candida albicans and non-albicans Candida in clinical samples and their intrinsic biofilm production status. Int J Med Sci Public Health. 2016;5(12)2443-2447.

doi:

[Article:https://doi.org/10.5455/ijmsph.2016.260420 16491][Crossref]

22. Rudrappa PT, Chandrashekar SC, Sumana MN. Speciation of Candida Isolates from Clinical Samples by Using Conventional and Chrom agar method. Int $\mathrm{J}$ Clin Microbiol App Sci. 2018;7(3)2663-2668.

doi:

[Article:https://doi.org/10.20546/ijcmas.2018.703.30 7][Crossref]

23. Kaur R, Dhakad MS, Goyal R, Kumar R. Emergence of non-albicans Candida species and antifungal resistance in intensive care unit patients. Asian Pacific J Trop Biomed. 2016;6(5)455-460.

doi:

[Article:http://dx.doi.org/10.1016/j.apjtb.2015.12.01 9][Crossref] 